Title:

\title{
Precise and Safe Landing Navigation Technologies for Solar System Exploration
}

Lead Author:

Dr. John M. Carson III

Space Technology Mission Directorate (STMD) Tech. Integration Mngr. - Precision Landing

National Aeronautics and Space Administration (NASA) Headquarters phone: (281) 483-1218, email: john.m.carson@nasa.gov

\section{Co-Authors:}

Ms. Michelle M. Munk ${ }^{++}$,

Mr. Ronald R. Sostaric,

Dr. Andrew E. Johnson,

Dr. Farzin Amzajerdian,

Mr. J. Bryan Blair,

Ms. Alicia Dwyer Cianciolo,

Dr. Larry H. Matthies,
NASA Headquarters

NASA Johnson Space Center

NASA Jet Propulsion Laboratory

NASA Langley Research Center

NASA Goddard Space Flight Center

NASA Langley Research Center

NASA Jet Propulsion Laboratory

Endorsements:

Mr. Bo J. Naasz ${ }^{\S}$,

Dr. Michael J. Wright ${ }^{\dagger}$, Mr. Gerald J. Lebeau ${ }^{\dagger}$, Dr. Ethiraj Venkatapathy ${ }^{\dagger}$, Mr. Michael J. Amato,
NASA Headquarters

NASA Ames Research Center

NASA Johnson Space Center

NASA Ames Research Center

NASA Goddard Space Flight Center

\footnotetext{
${ }^{\dagger}$ Member of NASA Entry, Descent and Landing (EDL) System Capabilities Leadership Team (SCLT)

${ }^{+}$Lead, NASA EDL SCLT and STMD EDL Principal Technologist

$\S$ Lead, NASA Rendezvous and Capture SCLT
} 


\begin{abstract}
Guidance, Navigation and Control (GN\&C) technologies for precise and safe landing are enablers for solar system exploration of destinations where terrain hazards or pre-positioned surface assets pose a significant risk to successful mission touchdown and surface operations. Technologies for precise landing enable intelligent descent maneuvers to minimize landed position error from targeted science locations and to avoid large terrain hazards (craters, hills, cliffs) viewable in a priori orbital reconnaissance maps. Technologies for safe landing enable intelligent landing-divert maneuvers to avoid smaller lander-relevant terrain hazards (rocks, boulders, sharp features) detectable in high-resolution, onboard descent imagery of much higher resolution than reconnaissance maps. These capabilities integrated onto a lander increase mission surface accessibility, reduce landing risks, increase mission science opportunities, and promote new mission concepts. Collectively, these capabilities are known within NASA as PL\&HA (Precision Landing and Hazard Avoidance) technology. PL\&HA has maintained consistent high prioritization within space technology roadmaps from NASA and the National Research Council (NRC) for more than a decade. NASA investments in PL\&HA have been ongoing since the mid 2000's and have involved funding from multiple mission directorates (STMD, SMD, HEO) and contributions from several centers and supporting institutions. The technologies are on track to be Technology Readiness Level (TRL) 8-9 between 2021-2024, with various component pieces being infused on the SMD Mars 2020 lander mission and into multiple SMD and STMD payloads on upcoming Commercial Lunar Payload Services (CLPS) missions.
\end{abstract}

Acronyms (quick reference for select acronyms)

$\begin{array}{llll}\text { EDL } & \text { Entry, Descent \& Landing } & \text { IMU } & \text { Inertial Measurement Unit } \\ \text { GN\&C } & \text { Guidance, Navigation \& Control } & \text { TRN } & \text { Terrain Relative Navigation } \\ \text { PL\&HA } & \text { Precision Landing \& Hazard Avoidance } & \text { HD } & \text { Hazard Detection }\end{array}$

\title{
1. Overview of Precise Safe Landing and Benefits to Exploration
}

Technologies for PL\&HA provide a spacecraft GN\&C subsystem with the knowledge necessary to plan and command maneuvers during descent and landing that enable a safe, precise and soft touchdown in close proximity to targeted surface science. The capabilities integrated onto a lander enable touchdown to within 10's to 100 meters of the targeted science site when integrated into a vehicle with sufficient propulsive and/or aerodynamic control authority to execute necessary diverts. Enabling a precise and safe touchdown increases mission surface accessibility and reduces landing risks, thereby promoting new mission science concepts and opportunities well beyond those feasible with current engineering capabilities. The PL\&HA technologies are extensible to landing on any solar system body with solid surfaces including the Moon, Mars, Europa, asteroids, comets, Icy Moons, and the solid surfaces on other Ocean Worlds. Because of this cross-cutting applicability, PL\&HA development has been a consistent priority within NASA and National Research Council (NRC) space technology roadmaps ${ }^{1,2}$ for more than a decade. The technologies are on track to achieve a TRL of 9 in the 2021-2024 timeframe, making them ready for infusion into the next decade of planetary science missions.

Precise landing refers to a minimization of landed position errors from targeted surface science locations, which could also include landing in close proximity to pre-positioned surface assets such as cached samples or other necessary mission components. Safe landing refers to spacecraft touchdown at surface locations where there is a minimum likelihood of small lander- 
relevant hazards, such as rocks, small boulders and sharp local slopes or features, being present that could pose a risk to either successful touchdown or subsequent surface operations. Precise landing contributes to safe landing when a priori orbital reconnaissance maps inform missions of large surface features, such as cliffs, hills, craters and large boulders, to avoid during landing operations. Soft landing refers to controlling spacecraft vertical and lateral velocities during final terminal descent and touchdown to minimize the impulsive force imparted to the vehicle during initial surface contact.

The operational regime for PL\&HA (Figure 1) is during the descent and landing portion of a mission Entry, Descent, and Landing (EDL) or Deorbit, Descent, and Landing (DDL). The EDL phase refers to landing on bodies with atmospheres, whereas DDL refers to landing on airless bodies; the acronym EDL will be used generically herein to represent either EDL or DDL. The PL\&HA technologies include multiple sensors, avionics and algorithms that together enable functions that include precise navigation, guidance planning, map-relative localization (also known as terrain relative navigation (TRN)), and surface hazard detection (HD) and avoidance. The multiple sensors used for these functions can include combinations of cameras, lidars, inertial measurement units (IMUs), velocimeters, and/or radars. The TRN function generates a global, precise position estimate to aid in determining guidance maneuvers for minimizing landed position error. The HD function analyzes high-resolution terrain images captured onboard during descent to detect small lander-relevant hazards and identify safe landing locations for final GN\&C divert maneuvers prior to touchdown. The PL\&HA sensors and functions can be leveraged individually or as an integrated system based on mission needs; when fully integrated, the technologies provide missions with the GN\&C capability for precise, safe and soft touchdown within 10's to 100 meters of targeted surface locations.

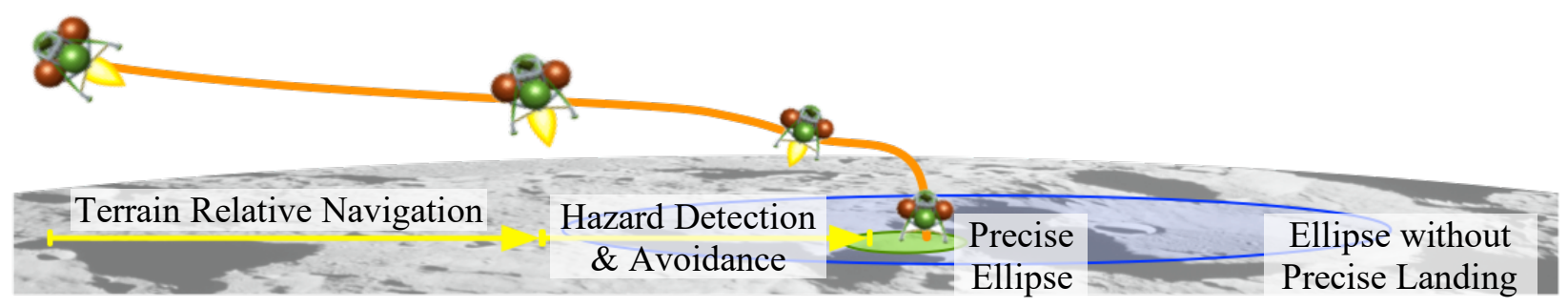

Figure 1. PL\&HA technologies operate during descent and landing following an entry/deorbit.

Investments in PL\&HA development and maturation have come through multiple NASA mission directorates including Space Technology Mission Directorate (STMD), Science Mission Directorate (SMD), and Human Explorations and Operations Mission Directorate (HEOMD) $)^{3,4,5,6}$. Multiple NASA centers and supporting institutions have been engaged in the development and mission infusion of the technologies, and commercialization of the technologies are in progress through multiple NASA public-private partnerships and technology licenses. Multiple current SMD missions including Mars 2020, as well as 2021 Commercial Lunar Payload Services (CLPS) missions are infusing components of PL\&HA technology. The technologies are also considered critical to upcoming Artemis human lunar missions, Mars Sample Return, Dragonfly, and with studies of a Europa Lander.

The data from PL\&HA is leveraged for onboard spacecraft GN\&C, but the data byproducts have other benefits to science and exploration. Specific data products include high-resolution images from three-dimensional point-cloud lidars and cameras, as well as precise surface-relative velocity and range measurements. The data sets have unprecedented spatial resolution along the 
descent ground track, decreasing to centimeter-level and smaller as the vehicle approaches landing (an order of magnitude finer than available from most orbiting assets). Swaths of subdecimeter resolution topography and imagery can provide a unique science perspective on, for example, boundary conditions for short-term surface dynamic processes, erosion, recent hypervelocity impact craters, and subtle sub-surface hazards. In addition, the sensor data is valuable to Plume-Surface Interaction (PSI) studies that could leverage the data to analyze surface material composition and plan future landing operations. The high-resolution terrain imagery also provides situational awareness for mission surface operations planning and roving.

\section{The Status Quo for Landing Versus Future Needs}

The status quo for landing missions, aside from Earth, is soft controlled touchdown. A typical Mars mission incorporates GN\&C sensors that include an IMU, plus radar-based range and velocity sensors ${ }^{7}$, which enable velocity- or range-based entry event triggers, followed by vertical and lateral velocity minimization during descent to soft touchdown. This approach has led to a history of successful NASA Mars landings, albeit with large landed uncertainty ellipses (Figure 2). The status quo provides no onboard, real-time knowledge of global surface position relative to targeted landing sites or knowledge of local terrain features upon terminal descent to landing - the EDL phase is and always has been blind in these respects (except for human landings of Apollo). Existing Mars landers also have limited propulsive control authority to execute large diverts to minimize these ellipses. Reconnaissance image resolution is typically sufficient to detect large terrain hazards but too coarse for direct detection of small lander-relevant hazards. This lack of detailed surface knowledge of small landerrelevant hazards, plus limited available spacecraft control authority, leads to mission site selection being a trade that balances scientific priorities versus engineering risks a trade that PL\&HA technologies help tip in

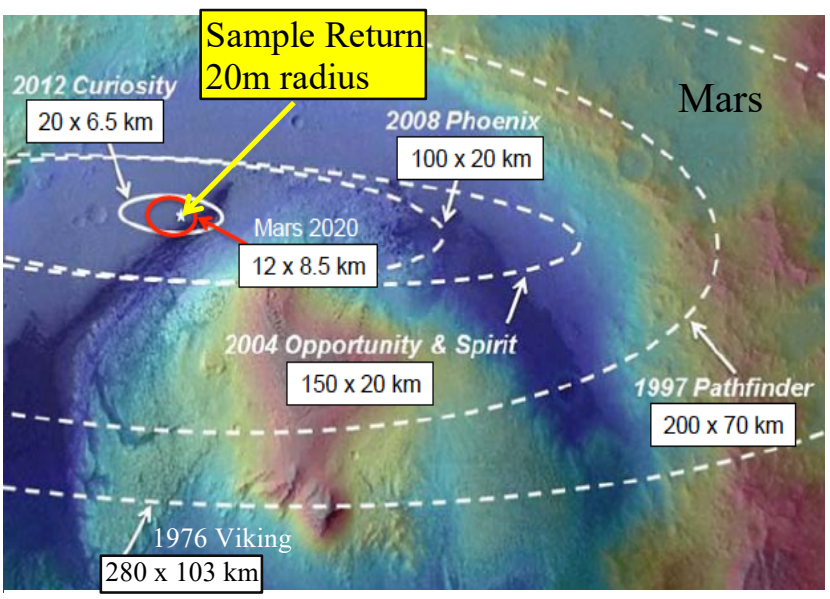

Figure 2. Overlay of NASA Mars landing ellipses (major \& minor axis dimensions shown) favor of scientific priorities.

The size of the landing ellipse is influenced by uncertainties in atmospheric entry conditions, vehicle aerodynamics, maneuvers, onboard sensor knowledge, and pre-EDL navigation. Through improvements in each of these areas, the current engineering capability for Mars landing is an ellipse on order of 5-km radius. For lunar landing the current capability is an approximate 500-m radius ellipse. To further reduce these ellipse sizes, precision landing technologies are required for providing global position information relative to the targeted science site, as well as sufficient spacecraft control authority for planning and executing large diverts. If sufficient control authority is available, the position estimate can be used to define a trajectory that will take the vehicle to a single specific landing site determined a priori - a pinpoint landing. If there is only limited onboard control authority, then the position estimate can still be used to reduce the landing ellipse, plus avoid any large hazards that were previously identified in reconnaissance maps of the landing area. For the Mars 2020 lander, which is NASA's first infusion of TRN into a planetary lander ${ }^{6}$, the position estimate and available 
spacecraft propulsive control authority will enable a divert of about 650 meters for touchdown within 60 -meters of surface locations (within the larger $12 \times 8.5 \mathrm{~km}$ ellipse) where previous analyses of Mars reconnaissance images indicate no large terrain hazards. For a future Mars Sample Return mission, the spacecraft will need to touch close to the pre-positioned sample caches - likely within a 20-meter landing ellipse pre-determined to be safe from Perseverance surface imaging. Achieving this considerable reduction in ellipse size requires increased spacecraft control authority to execute greater than $4 \mathrm{~km}$ large diverts, along with PL\&HA technology to provide global position awareness for planning the large diverts.

The multi-center NASA PL\&HA community is developing a knowledge base for integrating and evaluating the need (or not) for PL\&HA technologies within mission concepts. Multiple high-fidelity simulation toolsets enable detailed technical analysis of lander geometries and highfidelity simulations of EDL trajectories, PL\&HA sensor combinations and sensor qualities to study benefits of PL\&HA to missions. Lunar lander studies for both science and human missions to the southern lunar pole are investigating precise and safe landing capabilities on order of 50 meter radius ellipses and $10-\mathrm{cm}$ ground spatial resolution to enable landing on narrow, illuminated crater rims that have unknown size and distribution of rock ejecta (i.e., uncharacterized lander-relevant hazards). Europa Lander concept studies ${ }^{8}$ likewise indicate a need for onboard, high-resolution terrain imaging at $5-\mathrm{cm}$ ground spatial resolution to detect and avoid sharp ice penitents during landing. Avoidance of the hazards also requires precisely landing within the imaged region, which is a 50-m radius ellipse. The very hot, dense atmosphere on Venus makes it the hardest place in the solar system for actively guided safe and precise landing, but science goals to land in the rough highland terrain make a PL\&HA capability desirable. The Dragonfly mission also plans to leverage safe landing technology in the last kilometer of descent to avoid surface hazards and touch down on smooth terrain between sand dunes ${ }^{9}$. These mission studies highlight the future needs for PL\&HA implementation.

\section{PL\&HA Technologies and Maturity}

Multiple PL\&HA technologies are on track to achieve TRL 9 in the 2021-2024 timeframe, making them ready for infusion into the next decade of planetary science missions. These include camera-based TRN, Doppler lidar-based velocimetry, and lidar-based HD.

\subsection{Terrain Relative Navigation (or Map Relative Localization)}

TRN systems estimate position relative to an onboard stored map generated from orbital reconnaissance data taken before landing. This concept originated during the development of guided cruise missiles, but since then has been an active area of technology development within the planetary landing community. There are multiple different approaches to solving the map relative localization problem, but they all match sensor data collected on-board to the map generated before landing. Typically, due to their high angular resolution, visible cameras or lidars are used for these onboard sensor measurements.

The most mature TRN system for planetary EDL is the NASA Lander Vision System (LVS) for the Mars 2020 mission $^{5}$. The LVS estimates position with errors less than $40 \mathrm{~m}$ at $4 \mathrm{~km}$ altitude by fusing IMU data and landmark matches between descent images and a map (Figure $3)$. The LVS map is $6 \mathrm{~m} /$ pixel and carefully constructed to reduce local distortions $(<10 \mathrm{~m})$ and map-tie errors $(<100 \mathrm{~m})$ that will degrade position estimation accuracy. The LVS was TRL 8 at Mars 2020 launch and will be TRL 9 after Mars 2020 EDL in February 2021. 
The NASA OSIRIS-REx mission to asteroid Bennu also has a mature, commerciallydeveloped form of TRN for autonomous navigation that will be used for surface touch-and-go sampling in late $2020^{10}$. Multiple other commercial and non-profit entities are also developing TRN systems for robotic lunar landings in 2021 and 2022, which will bring them to TRL 8 or 9 depending on mission criticality (i.e., onboard demonstration vs inline operation, respectively).

Spaceflight TRN systems are enabling the next level of science return while the technology is also advancing in multiple new directions. Feature signatures that can be matched between map and image with little or no information about altitude and attitude are in development to create TRN systems that are less dependent on input from traditional navigation
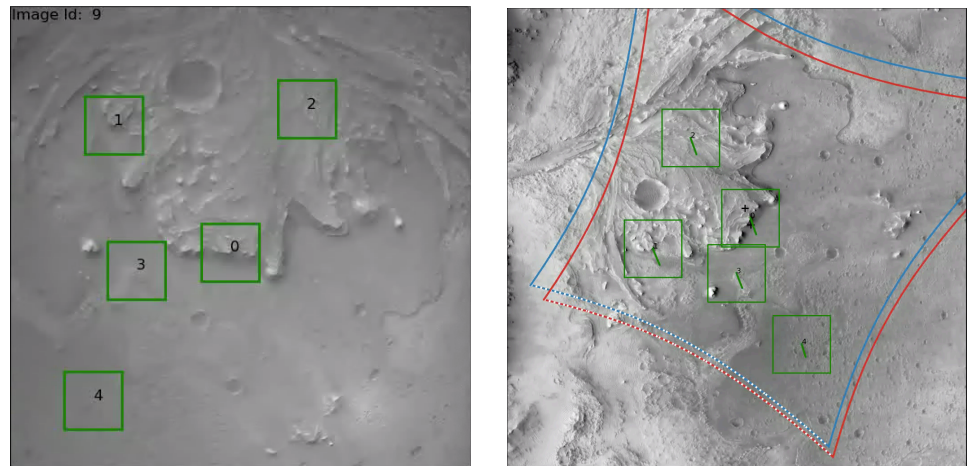

Figure 3. Landmark matches (green) in simulated Mars 2020 LVS landing images: camera (left) and map (right) sensors. This also includes development of methods to further integrate landmark matching directly into the navigation filter for improved estimation performance and stability. On-board simultaneous localization and mapping approaches are being investigated, which could reduce the need for a priori reconnaissance and shorten ground-based processing. Lidar-based TRN ${ }^{11}$, which enables landing in the dark or in poor lighting conditions, continues to gain traction especially with the recent push to land close to the lunar south pole. Finally, new techniques for generation and validation of high-resolution and accurate maps are in development to increase the accuracy of TRN position estimates.

\subsection{Doppler lidar-based Velocity and Range Sensing}

Directly measuring the Doppler frequency shift of light provides an extremely high-precision velocity measurement. A NASA technology called the Navigation Doppler Lidar (NDL), which has been in development since 2006, incorporates a customized laser waveform and optical homodyne detection to make velocity and range measurements. The NDL contains an electronics/photonics box and three fiber-coupled telescopes (Figure 4) that provide full vector velocity plus range measurements with precisions of $\sim 6 \mathrm{~cm} / \mathrm{sec}$ (3-sigma) and $\sim 6 \mathrm{~m}$ (3-sigma), respectively ${ }^{12}$. The technology is significantly smaller size/weight/power than similar Doppler radar systems (e.g., NDL occupies about 1/10 the volume), and the pencil-thin laser beams have very low beam divergence, which diversifies the telescope packaging and integration options for missions. NDL measurement precision is estimated to be more than an order of magnitude better than the Mars Terminal Descent System (TDS) Doppler radar ${ }^{7}$. The NDL technology has already passed the TRL 6 mark through NASA investments, and the sensor will achieve TRL 9 in 2021 with flights onboard two CLPS lunar landing missions.
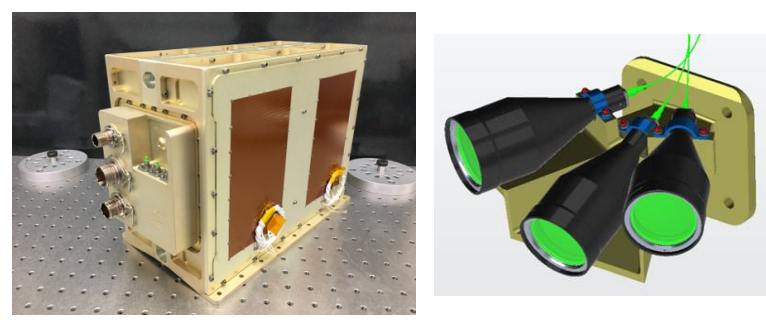

Figure 4. NASA NDL electronics/photonics box (left) and fiber-coupled telescopes (right) 
The NASA Doppler lidar technology has been licensed to a commercial vendor for development into a commercially available spaceflight sensor, with likely availability in the 2023 timeframe. In addition, a different commercial Doppler lidar sensor from the militaryaerospace commercial market has also flown in space. Terrestrial Doppler lidar sensors from the autonomous vehicles market are also being investigated for spaceflight maturation. This increasing commercial availability provides options to missions requiring high-precision velocimeters.

\subsection{Hazard Detection Sensors}

The detection of small lander-relevant hazards (rocks, small boulders, sharp features or excessive local slopes) cannot be accomplished with existing orbital reconnaissance maps, except possibly at Earth. Instead, onboard sensors are necessary to generate high-resolution images for onboard, real-time HD analysis to determine safe landing sites and subsequent divert maneuvers for safe touchdown. NASA has investigated multiple methods for HD, including lidars and cameras with accompanying algorithms. The most mature HD methods use active lidar-based sensing ${ }^{4,5}$ to generate three-dimensional range maps of illuminated, shadowed, and unlit terrain for direct detection of hazards. There are also passive camera-based methods ${ }^{13}$ that image illuminated terrain and infer hazard sizes based on detected shadow dimensions and knowledge of the local sun angle.

NASA has conducted multiple lidar-based HD studies for robotic and human lander mission concepts to different destinations, including an investigation of various lidar technologies that include scanning/scan-array $\operatorname{lidar}^{5}$ and flash lidar ${ }^{4}$ leveraging both analog detection and photon counting 8 techniques. The general results (e.g., Moon, Mars, Europa) indicate a need for maps with a 5-10 $\mathrm{cm}$ ground spatial resolution, captured from a range of $500 \mathrm{~m}$ to $1 \mathrm{~km}$, and with a range precision of $2-5 \mathrm{~cm}$ (1-sigma). There are not yet off-the-shelf available lidar that achieve these requirements, although some available commercial flash and scanning lidars could be suitable for hazard detection with some missions. NASA has invested significantly in TRL maturation for each of the various lidar technologies. A NASA scan-array lidar named the Hazard Detection Lidar (HDL) $)^{5}$ leverages spaceflight heritage components and is on track to be TRL 6 in 2022 and TRL 9 in 2023-2024 via an in-development lunar landing mission. Separate NASA investments in photon-counting lidar $^{8}$ targeted to the high-radiation environment of Europa are also on track for reaching TRL 6 in 2022.

The NASA HDL consists of an electronics/photonics box and a fiber coupled optical head (Figure 5) - the head includes a telescope and two spinning Risley prisms that are controlled to a specified scan pattern to maximize over-sampling of ground pixels to eliminate mapping gaps. When operating from a 500-meter range and a near-vertical descent, the HDL generates a 100meter-diameter circular map in 2 seconds with a 5 -cm ground sample distance and a range precision of $1 \mathrm{~cm}$ (1-sigma). The HDL can be operated at multiple ranges during descent for use in other PL\&HA functions such as early site evaluation and map updates to TRN. The surface data can also provide high-resolution maps and intensity images to inform mission science and plan ground operations.
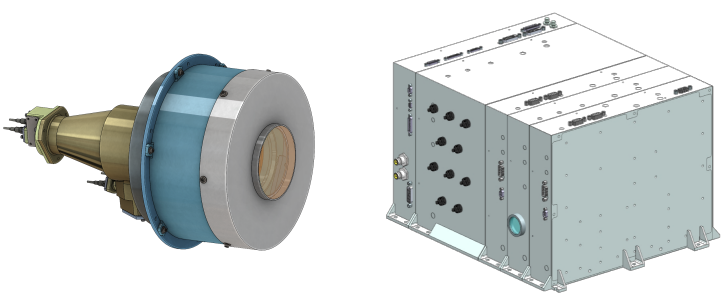

Figure 5. NASA HDL in-dev optical head (left) and electronics/photonics box (right) 


\section{Closing Remarks}

The status quo in engineering capabilities for landing result in large landing ellipses that limit surface accessibility and ultimately reduce mission science opportunities. With PL\&HA technologies and commensurate spacecraft control authority, a mission can touch down safely within 10 's to 100 meters of the targeted science site. This capability changes the conversation around landing site selection from one of engineering risk to one of maximizing science return. The PL\&HA technologies are all on track to be TRL 9 between 2021-2024, making them ready for mission infusion into the next decade of planetary science missions.

\section{References}

1. National Research Council of the National Academies, NASA Space Technology Roadmaps and Priorities: Restoring NASA's Technological Edge and Paving the Way for a New Era in Space, The National Academies Press, 2012.

2. Office of the Chief Technologist, 2015 NASA Technology Roadmaps, NASA, 2015.

3. C. D. Epp, T. B. Smith, "Autonomous Precision Landing and Hazard Detection and Avoidance Technology (ALHAT)," 2007 IEEE Aerospace Conference, doi: 10.1109/AERO.2007.352724.

4. C. Epp, E. Robertson, J. Carson, "Developing Autonomous Precision Landing and Hazard Avoidance Technology from Concepts through Terrestrially Flight-Tested Prototypes," AIAA GN\&C Conference, 2015, doi: 10.2514/6.2015-0324.

5. J. Carson, M. Munk, R. Sostaric, et al, "The SPLICE Project: Continuing NASA Development of GN\&C Technologies for Safe and Precise Landing," AIAA Scitech 2019 Forum, doi: 10.2514/6.2019-0660.

6. A. Johnson, et al, "The Lander Vision System for Mars 2020 Entry Descent and Landing," Proc. AAS GN\&C Conference (AAS-17-038), 2017.

7. C. Chen, B. Pollard, "Radar Terminal Descent Sensor Performance During Mars Science Laboratory Landing," Journal of Spacecraft and Rockets, 2014, doi: 10.2514/1.A32641.

8. A. San Martin, et al., "A Hazard Detection Sensor for Landing on Europa," NASA Active Optical Technical Interchange Meeting, 2018, uri: hdl.handle.net/2014/48609.

9. M. Wright, et al., "The Dragonfly Entry And Descent System," International Planetary Probes Workshop, presentation, 2019, uri: ntrs.nasa.gov/citations/20190028683.

10. C. Miller, et al., "On-Orbit Evaluation of Natural Feature Tracking for OSIRIS-REx Sample Collection," Proc. AAS GN\&C Conference, AAS-20-154, 2020.

11. A. Johnson, T. Ivanov, "Analysis and Testing of a LIDAR-Based Approach to Terrain Relative Navigation for Precise Lunar Landing," AIAA GN\&C Conference, 2011, doi: 10.2514/6.2011-6578.

12. F. Amzajerdian, et al., "Demonstration of coherent Doppler lidar for navigation in GPSdenied environments," SPIE Proceedings Volume 10191: Laser Radar Technology and Applications XXII, 2017, doi: 10.1117/12.2266972.

13. L. Matthies, et al., "Stereo Vision and Shadow Analysis for Landing Hazard Detection," 2008 IEEE International Conference on Robotics and Automation, doi: 10.1109/ROBOT.2008.4543625. 